\title{
A thermodynamically consistent model for electric double layers in Li-all-solid-state batteries
}

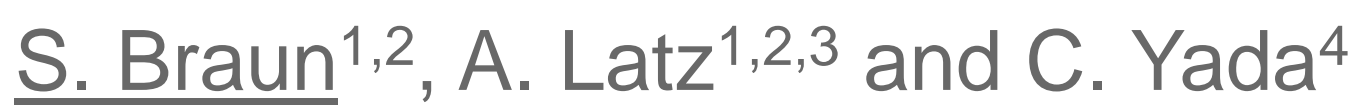 \\ ${ }^{1}$ German Aerospace Center, Stuttgart, Germany \\ 2 Helmholtz Institute for Battery Research UIm, UIm, Germany \\ 3 UIm University, Ulm, Germany \\ 4 Toyota Motor Europe NVISA, Zaventem, Belgium
}

November $27^{\text {th }}, 2014$

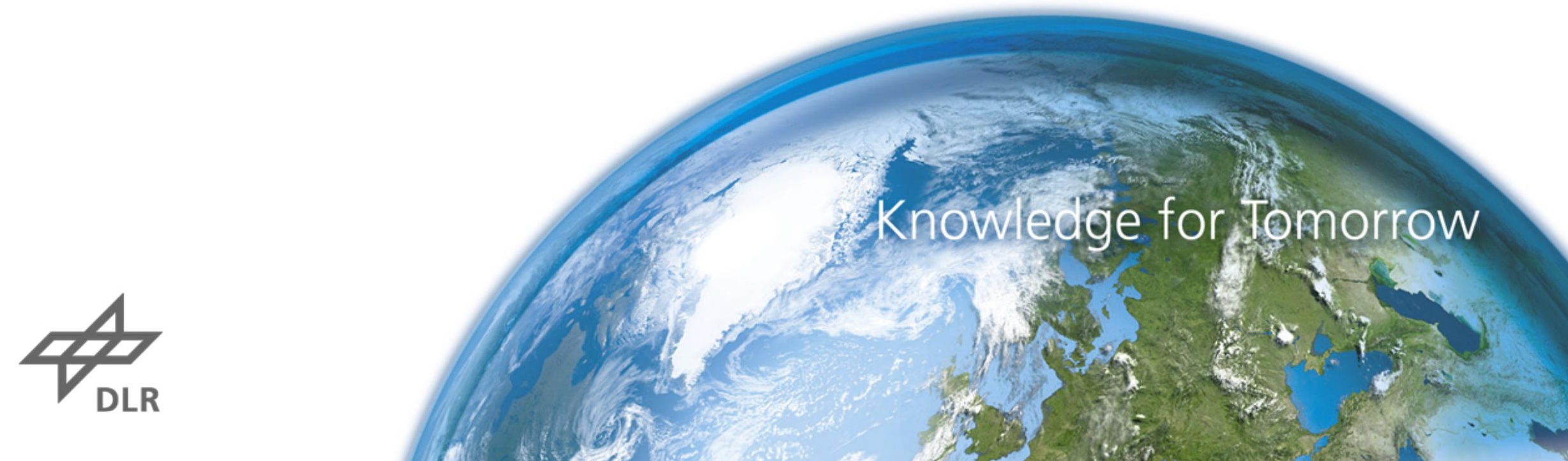




\section{Outline}

- Motivation: Lithium-deficient layers in solid electrolytes

- The mathematical model

- General properties

- 1D stationary boundary value problem

- Results: Cation and potential distributions

- Conclusions \& Outlook 


\section{Motivation:}

\section{Lithium-deficient layers (LDLs) in solid electrolytes}

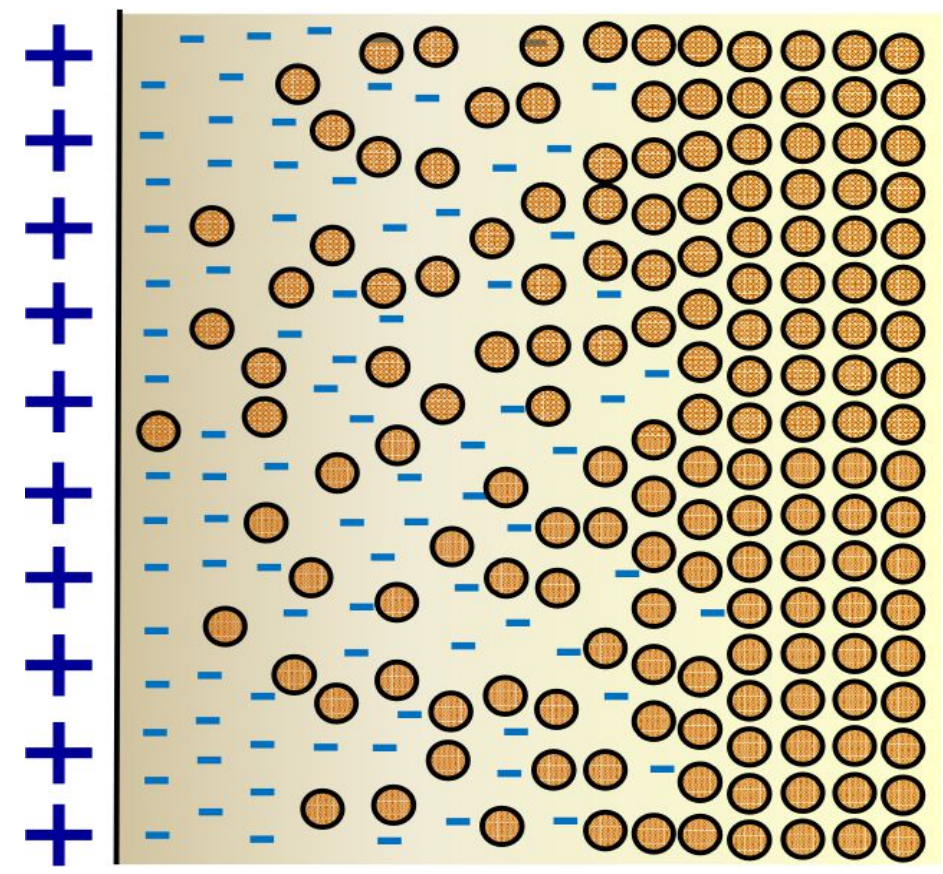

Applied external el. potential:

- Anode:

- Li ions vacate region near electrode

- Area partially or totally void of Li ions develops (LDL)

- Cathode:

- Vacancies fill up with Li ions

- Area with few or no vacancies develops

- In space charge regions:

high electric fields, reduced conductivity Limits the performance of the battery!

Numerical study: Ideas for improved battery concepts leading to higher power density? 


\section{Properties of the model (I)}

What properties should the model have?

- As general as possible (e.g., material independent)

- Usable in a full-cell model

- Easily extendable to include more physics

- Accurately describe dynamics inside LDL

- Consistent with laws of physics and thermodynamics [1]! 


\section{Properties of the model (II)}

\section{How is the model realised mathematically/physically?}

- Li ions and vacancies modeled as a continuum

- Simplifications:

- Isothermal condition

- Only one type of vacancies

- Based on first principles only:

- Mass conservation:

$$
\partial_{t} \rho_{\alpha}+\nabla \cdot\left(\rho_{\alpha} v_{\alpha}\right)=0
$$

- momentum conservation:

$$
\rho \dot{\boldsymbol{v}}=\nabla \cdot \boldsymbol{T}+\rho \boldsymbol{b}
$$

- Energy conservation: $\rho \dot{\epsilon}=\nabla\left(\boldsymbol{T}^{T} \boldsymbol{v}\right)+\rho \boldsymbol{v} \cdot \boldsymbol{b}-\nabla \cdot(\boldsymbol{q}-\boldsymbol{E} \times(\boldsymbol{v} \times \boldsymbol{D}))+\rho h$

- Entropy inequality:

$$
\rho \dot{s}+\nabla \cdot J_{s}-\rho \frac{h}{\vartheta} \geq 0
$$

- Free-energy functional constitutes closure of the system:

$$
\rho \psi=\rho \psi_{R}+\rho \psi_{p}+\rho \psi_{p o l}+k_{B} \vartheta\left(n_{c} \ln \left(\frac{n_{c}}{v}\right)+\left(v-n_{c}\right) \ln \left(\frac{v-n_{c}}{v}\right)\right)
$$




\section{Properties of the model (II)}

\section{How is the model realised mathematically/physically?}

- Li ions and vacancies modeled as a continuum

- Simplifications:

- Isothermal condition

- Only one type of vacancies

- Based on first principles only:

- Mass conservation:

$$
\partial_{t} \rho_{\alpha}+\nabla \cdot\left(\rho_{\alpha} \boldsymbol{v}_{\alpha}\right)=0
$$

- momentum conservation:

$$
\rho \dot{\boldsymbol{v}}=\nabla \cdot \boldsymbol{T}+\rho \boldsymbol{b}
$$

- Energy conservation: $\rho \dot{\epsilon}=\nabla\left(\boldsymbol{T}^{T} \boldsymbol{v}\right)+\rho \boldsymbol{v} \cdot \boldsymbol{b}-\nabla \cdot(\boldsymbol{q}-\boldsymbol{E} \times(\boldsymbol{v} \times \boldsymbol{D}))+\rho h$

- Entropy inequality:

$$
\rho \dot{s}+\nabla \cdot J_{s}-\rho \frac{h}{\vartheta} \geq 0
$$

Li ion hopping

- Free-energy functional constitutes closure of the system:

$$
\rho \psi=\rho \psi_{R}+\rho \psi_{p}+\rho \psi_{p o l}+k_{B} \vartheta\left(n_{c} \ln \left(\frac{n_{c}}{v}\right)+\left(v-n_{c}\right) \ln \left(\frac{v-n_{c}}{v}\right)\right)
$$




\section{Properties of the model (II)}

\section{How is the model realised mathematically/physically?}

- Li ions and vacancies modeled as a continuum

- Simplifications:

- Isothermal condition

- Only one type of vacancies

- Based on first principles only:

- Mass conservation:

- momentum conservation:

- Energy conservation: $\rho \dot{\epsilon}=\nabla\left(\boldsymbol{T}^{T} \boldsymbol{v}\right)+\rho \boldsymbol{v} \cdot \boldsymbol{b}-\nabla \cdot(\boldsymbol{q}-\boldsymbol{E} \times(\boldsymbol{v} \times \boldsymbol{D}))+\rho h$

- Entropy inequality:

$q$... heat flux

$$
\partial_{t} \rho_{\alpha}+\nabla \cdot\left(\rho_{\alpha} \boldsymbol{v}_{\alpha}\right)=0 \text { Thermodynamic }
$$$$
\rho \dot{\boldsymbol{v}}=\nabla \cdot \boldsymbol{T}+\rho \boldsymbol{b} \quad \text { consistency! }
$$

$$
\rho \dot{s}+\nabla \cdot J_{s}-\rho \frac{h}{\vartheta} \geq 0
$$

$\rho$... mass density

$\alpha$... particle index

$v$... velocity

$\boldsymbol{T}$... stress tensor

b ... body force

$\epsilon$... energy density

$\boldsymbol{E}$... electric field

D ... electric displacement

$h$... body heat

$s$... entropy density

$J_{S} \ldots$ entropy flux

$\vartheta$... temperature

$k_{B} \quad \ldots$ Boltzmann constant

$n_{\alpha} \ldots$ density of particle $\alpha$

$v=1-n_{a}$

Li ion hopping

- Free-energy functional constitutes closure of the system:

$$
\rho \psi=\rho \psi_{R}+\rho \psi_{p}+\rho \psi_{p o l}+k_{B} \vartheta\left(n_{c} \ln \left(\frac{n_{c}}{v}\right)+\left(v-n_{c}\right) \ln \left(\frac{v-n_{c}}{v}\right)\right)
$$




\section{D stationary boundary value problem}

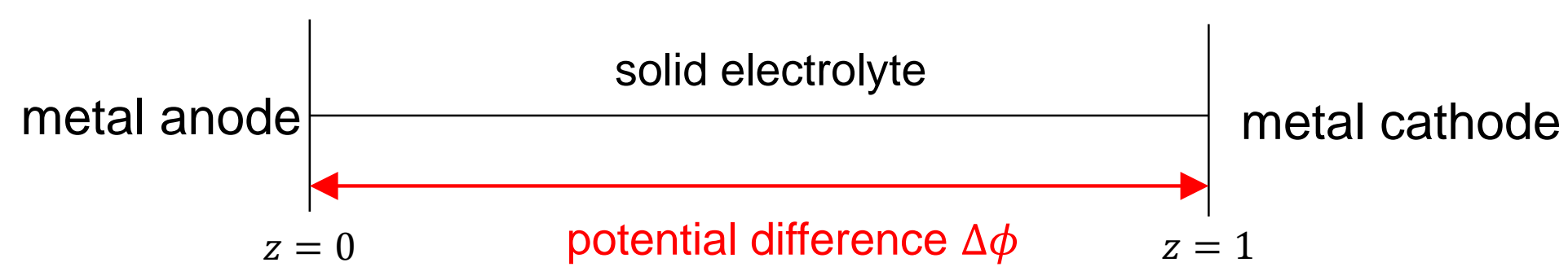

Set of equations:

$$
\begin{gathered}
\partial_{z}\left(\mu_{c}-\frac{m_{c}}{m_{a}}\left(\mu_{a}-\frac{v}{n_{a}} \mu_{v}\right)\right)+\left(z_{c}-\frac{m_{c}}{m_{a}} z_{a}\right) \partial_{z} \phi=0 \\
a^{2} \partial_{z} p=-n^{F} \partial_{z} \phi \\
\lambda^{2} \partial_{z z} \phi=-n^{F}
\end{gathered}
$$

$\mu_{\alpha}$... chemical potential

$n^{F}$... charge density

$\phi, p$... el. potential and pressure

$z_{\alpha}, m_{\alpha} \ldots$ particle charge and mass

$a, \lambda, \epsilon \ldots$ parameters

$F\left(n^{F}\right), H\left(n^{F}\right) \ldots$ coefficient functions 


\section{D stationary boundary value problem}

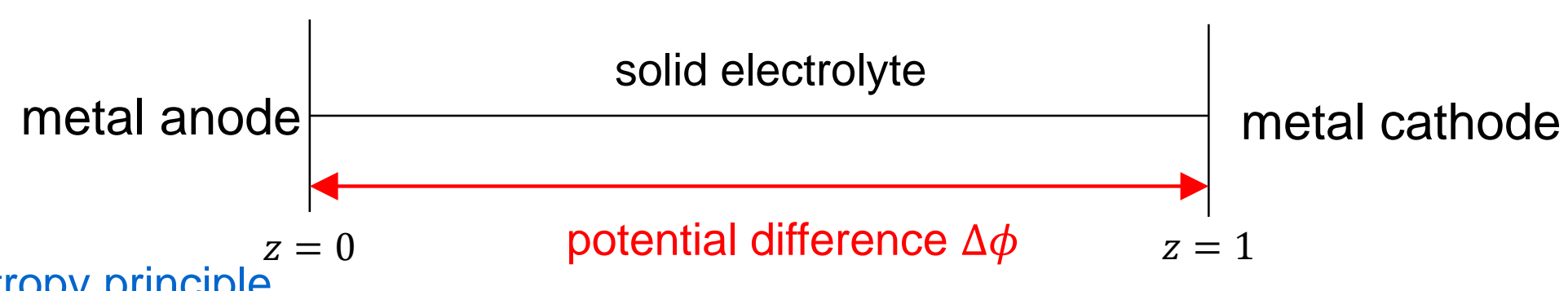

from entropy principle

$$
\begin{gathered}
\downarrow \frac{\text { Set of equations: }}{\partial_{z}\left(\mu_{c}-\frac{m_{c}}{m_{a}}\left(\mu_{a}-\frac{v}{n_{a}} \mu_{v}\right)\right)+\left(z_{c}-\frac{m_{c}}{m_{a}} z_{a}\right) \partial_{z} \phi=0} \\
a^{2} \partial_{z} p=-n^{F} \partial_{z} \phi \\
\lambda^{2} \partial_{z z} \phi=-n^{F}
\end{gathered}
$$

$\mu_{\alpha} \ldots$ chemical potential

$n^{F}$... charge density

$\phi, p$... el. potential and pressure $z_{\alpha}, m_{\alpha} \ldots$ particle charge and mass

$a, \lambda, \epsilon \ldots$ parameters

$F\left(n^{F}\right), H\left(n^{F}\right)$... coefficient functions 


\section{D stationary boundary value problem}

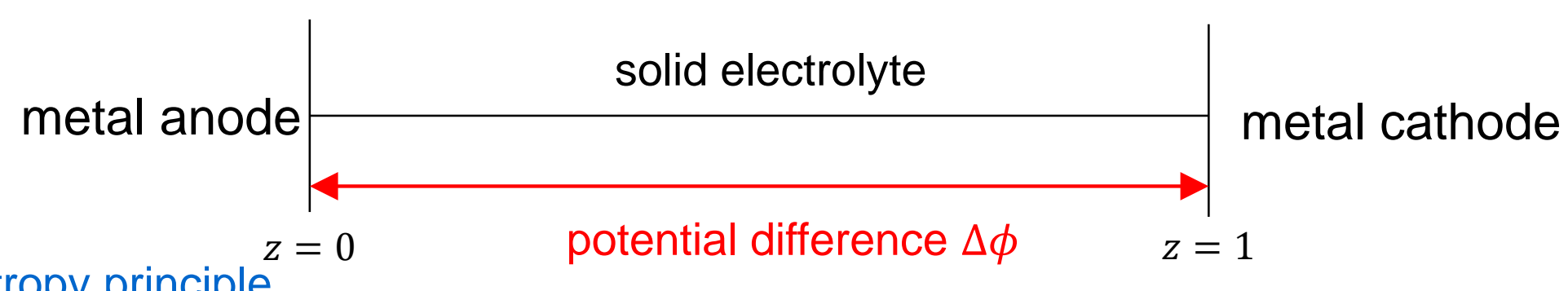

from entropy principle

$$
\begin{aligned}
& \downarrow \quad \text { Set of equations: } \\
& \partial_{z}\left(\mu_{c}-\frac{m_{c}}{m_{a}}\left(\mu_{a}-\frac{v}{n_{a}} \mu_{v}\right)\right)+\left(z_{c}-\frac{m_{c}}{m_{a}} z_{a}\right) \partial_{z} \phi=0 \\
& a^{2} \partial_{z} p=-n^{F} \partial_{z} \phi<\text { momentum } \\
& \lambda^{2} \partial_{z z} \phi=-n^{F} \quad \text { balance }
\end{aligned}
$$

$\mu_{\alpha} \ldots$...chemical potential

$n^{F}$... charge density

$\phi, p \quad \ldots$ el. potential and pressure

$z_{\alpha}, m_{\alpha} \ldots$ particle charge and mass

$a, \lambda, \epsilon \ldots$ parameters

$F\left(n^{F}\right), H\left(n^{F}\right) \ldots$ coefficient functions 


\section{D stationary boundary value problem}

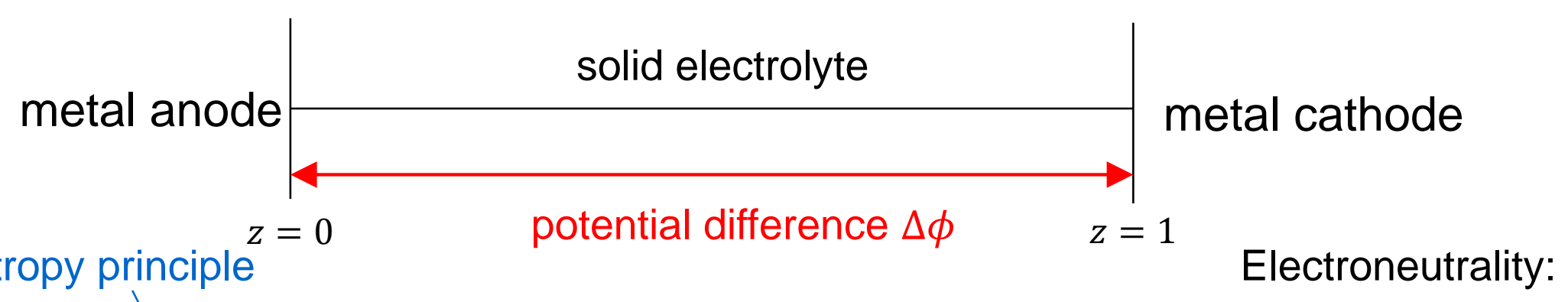

Set of equations:

$$
\begin{aligned}
& \frac{\text { Set of equations: }}{\partial_{z}\left(\mu_{c}-\frac{m_{c}}{m_{a}}\left(\mu_{a}-\frac{v}{n_{a}} \mu_{v}\right)\right)+\left(z_{c}-\frac{m_{c}}{m_{a}} z_{a}\right) \partial_{z} \phi=0} \int_{0}^{1} n^{F} d z=0 \\
& \begin{array}{l}
\text { Poisson } \\
\text { equation }
\end{array} a^{2} \partial_{z} p=-n^{F} \partial_{z} \phi<\begin{array}{l}
\text { momentum } \\
\text { balance }
\end{array} \\
& z=\int_{n_{0}^{F}}^{n_{1}^{F}} \frac{F\left(\widetilde{n}^{F}\right) d \widetilde{n}^{F}}{\sqrt{\epsilon+2 / \lambda^{2}\left(H(0)-H\left(\widetilde{n}^{F}\right)\right)}} \\
& \phi(z=0)=\phi_{L} ; \phi(z=1)=\phi_{R} \\
& p(z=0)=p_{0}
\end{aligned}
$$

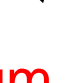




\section{Results (I): Cation and potential distributions}
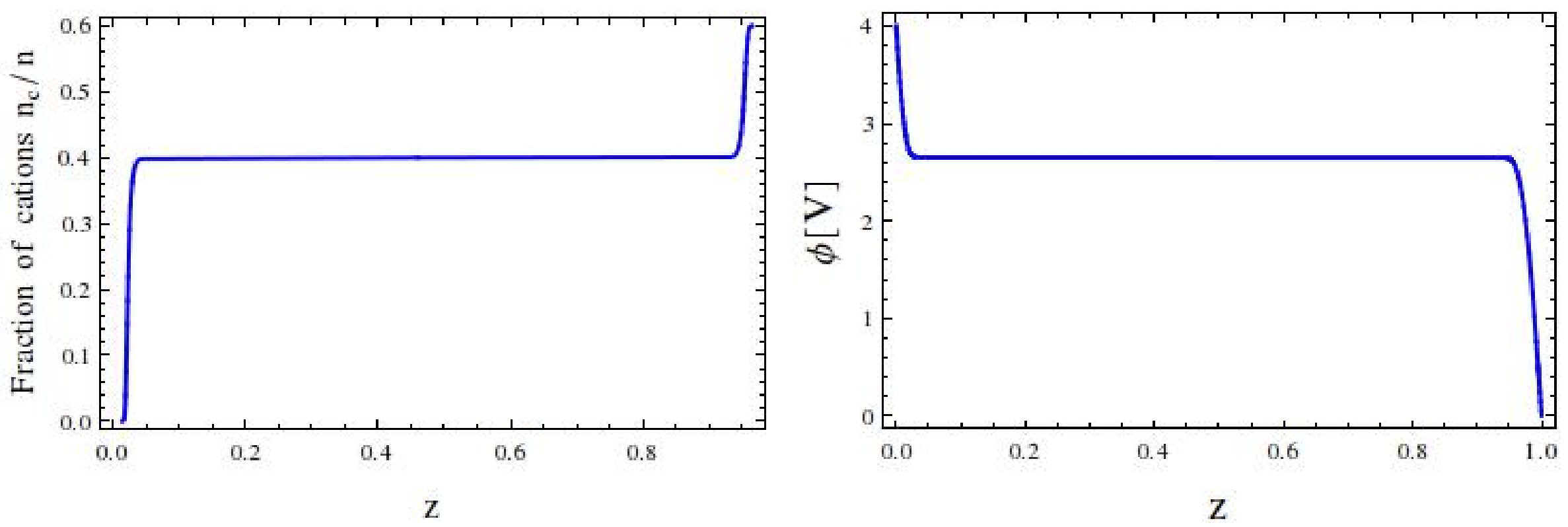

- Potential variation on same length scale as density variation

- $\mathrm{Li}_{1+\mathrm{x}+3 \mathrm{z}} \mathrm{Al}_{\mathrm{x}}(\mathrm{Ti}, \mathrm{Ge})_{2-\mathrm{x}} \mathrm{Si}_{3 \mathrm{z}} \mathrm{P}_{3-\mathrm{z}} \mathrm{O}_{12}$ [1] at 4.0V: space charge layers $\sim 400-600 \mathrm{~nm}$

Classical PNP-theory:

LDL $\sim 1-10 \AA$
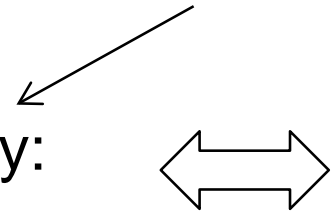

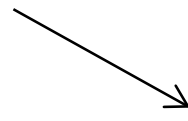

Experiments by Yamamoto et al [2]:

$\mathrm{LDL} \sim 1 \mu \mathrm{m}\left(\right.$ for $\mathrm{Li}_{1+\mathrm{x}+\mathrm{y}} \mathrm{Al}_{\mathrm{y}} \mathrm{Ti}_{2-\mathrm{y}} \mathrm{Si}_{\mathrm{x}} \mathrm{P}_{3-\mathrm{x}} \mathrm{O}_{12}$ )

[1] Courtesy Y. Inda, Ohara Inc. [2] K. Yamamoto et al, Angew. Chem. Int. Ed. 49, 4414-4417 (2010) 


\section{Results (II): different electrode potentials}
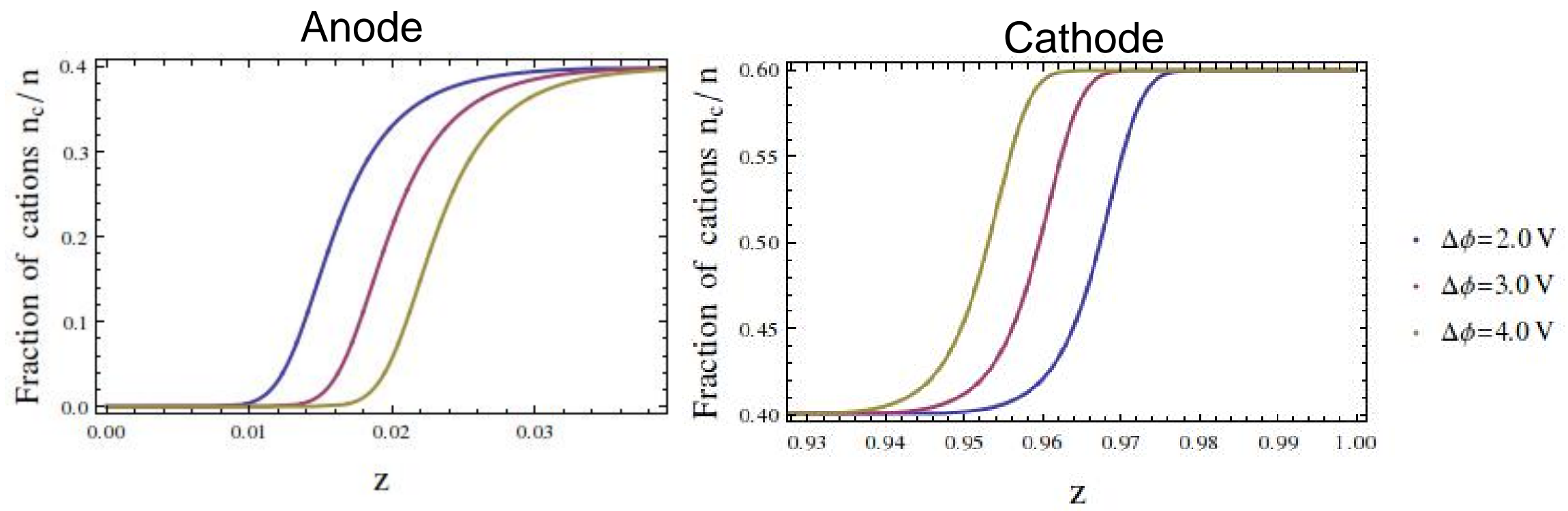

$\mathrm{Li}_{1+\mathrm{x}+3 \mathrm{z}} \mathrm{Al}_{\mathrm{x}}(\mathrm{Ti}, \mathrm{Ge})_{2-\mathrm{x}} \mathrm{Si}_{3 \mathrm{z}} \mathrm{P}_{3-\mathrm{z}} \mathrm{O}_{12}:$

- electrode potentials were varied between $\Delta \phi=2.0 \mathrm{~V}, 3.0 \mathrm{~V}, 4.0 \mathrm{~V}$

- Changes of the order of a factor $\sim 2$ in a real battery possible 


\section{Results (III): different material parameters (varying $\lambda$ )}

Anode

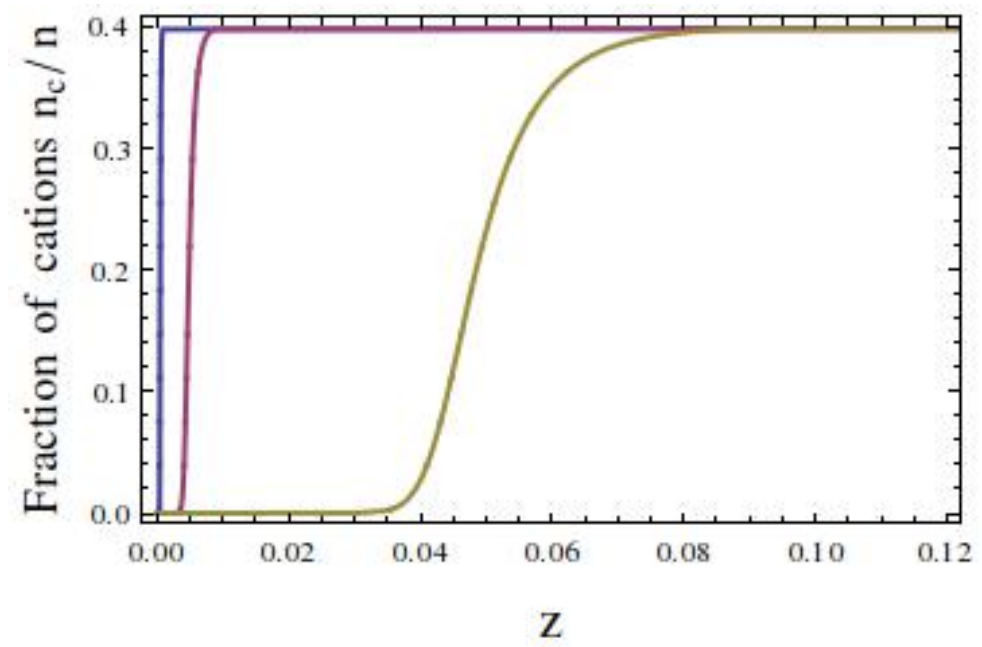

- $\lambda=\sqrt{\frac{k_{B} T \epsilon_{0}(1+\chi)}{n_{R} e_{0}^{2} L_{R}^{2}}} \sim \frac{\text { Debye length }}{\text { system length }}$
Cathode

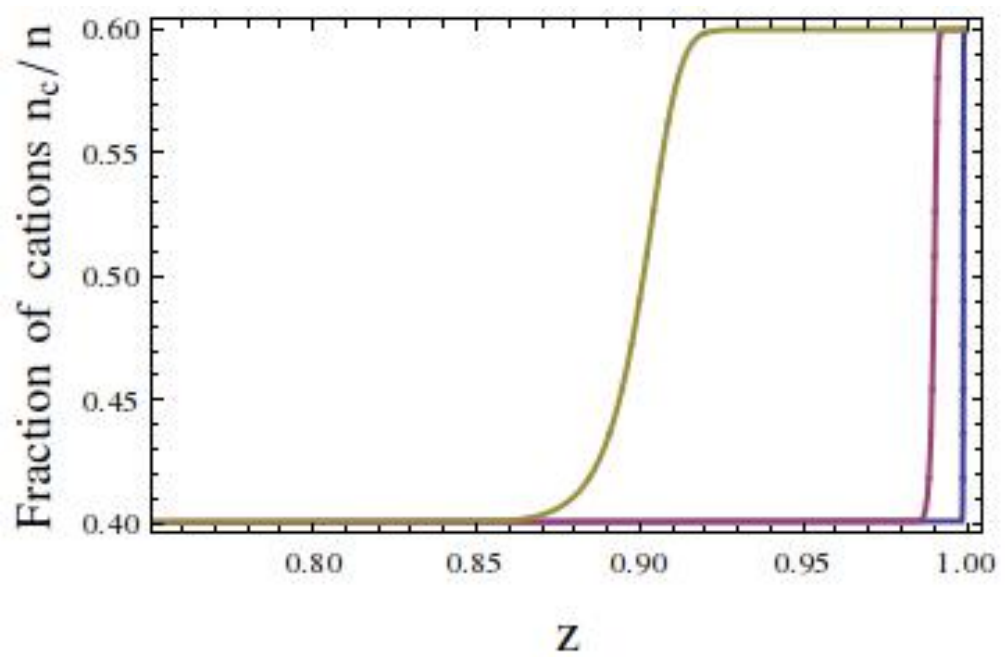

$\lambda=3.16 \mathrm{e}-5$

- $\lambda=3.16 \mathrm{e}-4$

- $\lambda=3.16 \mathrm{e}-3$

- $\lambda$ mainly determined by dielectric properties of the material $(\chi)$ ( $\chi$ can differ by orders of magnitude! $\mathrm{La}_{0.5} \mathrm{Li}_{0.5} \mathrm{TiO}_{3}: \chi \sim 10^{5}, \mathrm{LiPO}_{3}: \chi \sim 10^{2}$ )

- $\mathrm{LDL} \sim 10 \lambda$ (in a fluid: $\sim \lambda$ !)

$\square$ Dielectric properties are main influencing factor for LDL! 


\section{Conclusions}

- A thermodynamically consistent model for ion transport in a solid electrolyte was derived

- Predictions for the width of the LDL are roughly of the same order of magnitude as experimental findings for similar parameters

- Main influencing factor are the dielectric properties, external potential difference and other factors plays only minor role

\section{Outlook}

- Numerical implementation for solution of full time-dependent problem under way

- Possibility to "tailor" materials regarding dielectric properties

\section{Thank you!}

